\title{
Predicting periodontitis progression?
}

\section{Abstracted from}

\section{Lang NP, Suvan JE, Tonetti MS.}

Risk factor assessment tools for the prevention of periodontitis progression: a systematic review. / Clin Periodontol 2015; 42: S59-S70. doi: 10.1111/jcpe.12350

Address for correspondence: Niklaus P Lang, Scheuermattweg 33, CH-3043,

Uettligen, Switzerland. E-mail: nplang@switzerland.net

\section{Questions: What are the characteristics of currently published patient-based tools or systems used to assess levels of risk for periodontitis progression? Are results from current patient-based risk assessment tools predictive of periodontitis progression in adults treated for this disease?}

Data sources Cochrane Library, Ovid, Medline, Embase and LILACS were searched using no language restrictions and included information up to July 2014. Bibliographic references of included articles and related review articles were hand searched. On-line hand searching of recent issues of key periodontal journals was performed (Journal of Clinical Periodontology, Journal of Dental Research, Journal of Periodontal Research, Journal of Periodontology, Oral Health and Preventive Dentistry).

Study selection Prospective and retrospective cohort studies were used for answering the question of prediction since there were no randomised controlled trials on this topic. Risk of bias was assessed using the validated Newcastle-Ottawa quality assessment scale for non-randomised studies. Cross-sectional studies were included in the summary of currently reported risk assessment tools but not for risk of progression of disease, due to the inability to properly assess bias in these types of studies. Titles and abstracts were scanned by two reviewers independently.

Full reports were obtained for those articles meeting inclusion criteria or those with insufficient information in the title to make a decision. Any published risk assessment tool was considered. The tool was defined to include any composite measure of patient-level risk directed towards determining the probability for further disease progression in adults with periodontitis. Periodontitis was defined to include both chronic and aggressive forms in the adult population. Outcomes included changes in attachment levels and/or deepening of periodontal pockets in millimeters in study populations undergoing supportive periodontal therapy.

Data extraction and synthesis Data extraction was performed independently and in collaboration by two reviewers; completed evidence tables were reviewed by three reviewers. Studies were each given a descriptive summary to assess the quantity of data as well as further assessment of study variations within study characteristics. This also allowed for determining the suitability of data for further quantitative analysis (meta-analysis). Unfortunately, the heterogeneity of the data did not allow.
Results After screening, 19 studies fitted the inclusion criteria of identifying five different patient-based periodontal risk assessment tools. DenPlan Excel/Previsor Patient Assessment (DEP-PA) and its modifications were used in five studies. The HIDEP model, the dentition risk system (DRS) and the risk assessment-based individualised treatment (RABIT) were each used in one study. Lastly, the periodontal risk assessment (PRA) and its modifications were found in 12 publications.

PRA uses the following factors to assess risk of recurrence of disease: Percentage of bleeding on probing, loss of teeth from a total of 28 teeth, loss of periodontal support in relation to the patient's age, prevalence of residual pockets greater than $4 \mathrm{~mm}$ ( $3-5 \mathrm{~mm}$ ), systemic and genetic conditions and environmental factors, such as cigarette smoking.

Ten included studies had cohort designs ( $N=2130)$ spanning three to 12 years with different follow-up times. Generally, these studies reflected that different assessment tools were able to separate subjects with differing probability of disease progression and tooth loss. The observed effect was dose dependent (the higher the estimation of risk the higher the level of observed disease or tooth loss).

Six cross sectional studies ( $\mathrm{N}=1078)$ reported the comparison of different assessment tools, adjusted or unadjusted associations with periodontal disease and subjective risk assessments provided by the tools. There were three articles noted in the flow diagram as articles proposing the tool. Qualitative analysis reflects that parameters are similar across the studies but differences are present in how these parameters were assessed.

Conclusions In treated populations, results of patient-based risk assessments predicted periodontitis progression and tooth loss in various populations. Additional research on the utility of risk assessment and results in improving patient management are needed.

\section{Commentary}

Periodontal disease is a progressive disease that affects $50 \%$ of adults over the age of 30 and that number increases with age. The ability to develop valid tools that would assess a patient's risk of periodontal disease progression would be advantageous in the attempt to minimise its effects over an at-risk patient's lifetime.

Periodontal risk is a multi-faceted disease whose progression can be impacted by localised environment as well as genetics. An extensive search was performed using an appropriate number of databases and publications without language or year restrictions.

The authors wanted to search RCTs but evidence was unavailable so prospective and retrospective cohort studies were included as 
best available evidence. Results were not combined in meta-analysis as there was too much heterogeneity amongst the studies. Crosssectional studies were only included for assessment of tools.

The authors' goals were two-fold: to search out the available tools for assessing the risk of progression of periodontitis and tooth loss and to assess whether these tools are predictive of the progression of periodontitis.

They identified five tools: DEP-PA(PRC), HIDEP, RABIT, DRS and PRA.

DRS is a web-based analytical tool that calculates chronic peridontal risk for the dentition and if risk is found, prognosticates disease progression tooth by tooth.

HIDEP is computerised tool that uses predefined risk groups for selecting and managing individual treatment. It also assesses the risk of other aspects of oral health in addition to periodontal status.

RABIT uses a modified approach that supports individualised risk-based recall schedules during active therapy and the course of treatment.

DEP-PA(PRC) is also computer-based and is periodontal risk assessment focused. PRA is a functional diagram based upon the combination of various parameters that impact patient risk. PRA is similar but includes other factors like socio-economics and stress as factors.

Results were expressed in different ways depending on which tool was used - risk scores, tooth loss and risk factors. There were three articles noted in the flow diagram as articles proposing a tool but no mention was made of them as part of the discussion.

One of the studies used untreated patients but the question of this systematic review was meant to include treated patients. Results in some studies were expressed as tooth loss $=.5$, but from a clinical perspective is impossible to measure half a tooth. Another study's conclusions were that high-risk patients showed increased tooth loss and less bone fill than those at low or moderate risk which is information already known.

This systematic review was supported by the Clinical Research Foundation (CRF) for the Promotion of Oral Health (Switzerland) and the European Research Group on Periodontology (Italy). Although the authors state there was no conflict of interest, two of the authors developed the PRA for the progression of periodontitis after active therapy. They received no compensation and it is available free to anyone (www.perio-tools.com/PRA).

Precision of the results was not qualitatively assessed. The authors concluded that there are tools that successfully predict progression of periodontitis or tooth loss in various populations. As far as applicability to a patient population, the authors claim no studies are available to show how using a risk assessment may impact patient care.

This systematic review provided a thorough approach to defining the periodontal risk assessment tools that are available to the clinician. Although any of these tools may be applied to clinical settings, there is limited evidence as to their impact, long term, on the efficacy of assessing patient risk. Common sense allows for estimating risk assessment based on tooth loss for patients. More detailed longitudinal studies are needed for each of these tools to define which is most applicable to the oral health professional. Despite the authors' claim that PRC and PRA are the best tools, the evidence does not necessarily indicate which of the tools is 'best'. Also, there is the potential for a conflict of interests since two of the authors developed the PRA tool.

Debra M Ferraiolo New York University College of Dentistry, New York, USA

Evidence-Based Dentistry (2016) 17, 19-20. doi:10.1038/sj.ebd.6401152 